\title{
RELIABILITY ASSESSMENT OF SYSTEM UNDER A GENERALIZED RUN SHOCK MODEL
}

\author{
MIN GONG, * University of Science and Technology of China and \\ City University of Hong Kong \\ MIN XIE, ${ }^{* *}$ City University of Hong Kong \\ YANING YANG, ${ }^{* * *}$ University of Science and Technology of China
}

\begin{abstract}
In this paper we are concerned with modelling the reliability of a system subject to external shocks. In a run shock model, the system fails when a sequence of shocks above a threshold arrive in succession. Nevertheless, using a single threshold to measure the severity of a shock is too critical in real practice. To this end, we develop a generalized run shock model with two thresholds. We employ a phase-type distribution to model the damage size and the inter-arrival time of shocks, which is highly versatile and may be used to model many quantitative features of random phenomenon. Furthermore, we use the Markovian property to construct a multi-state system which degrades with the arrival of shocks. We also provide a numerical example to illustrate our results.
\end{abstract}

Keywords: Run shock model; phase-type distribution; Markov chain; reliability function; inter-arrival time; mean residual life

2010 Mathematics Subject Classification: Primary 93A30

Secondary $60 \mathrm{~J} 28$

\section{Introduction}

Reliability modelling is an important issue in probability theory and industrial engineering, aiming at predicting the lifetime behaviour of a system. The main factors that may affect the lifetime behaviour of a system usually consist of internal degradation and other external factors, such as random shocks or even intentional attacks [10], [14], [18]. A variety of shock models have been developed to study how external shocks act on a system. Generally speaking, shock models can be classified into five groups: cumulative shock model, extreme shock model, run shock model, $\delta$-shock model, and mixed shock model. For example, in a cumulative shock model, the system fails as soon as the cumulative magnitude of shocks exceeds a given level [7], while in a run shock model, the system is considered failed when there is a series of shocks whose magnitude is greater than a threshold. Besides, the mixed shock model is a combination of at least two types of different model. For example, Cha et al. [1] extended the results in an extreme shock model by combining it with a cumulative shock model. Some other examples can be found in [8] and [11].

\footnotetext{
Received 2 February 2018; revision received 1 October 2018.

* Postal address: Shenzhen Research Institute, City University of Hong Kong, 8 Yuexing First Road, Shenzhen, Guangdong, China. Email address: mingong3-c@my.cityu.edu.hk

** Postal address: Department of Systems Engineering and Engineering Management, City University of Hong Kong, Tat Chee Avenue, Hong Kong, China. Email address: minxie@ cityu.edu.hk

*** Postal address: Department of Statistics and Finance, University of Science and Technology of China, 96 Jinzhai Road, Hefei, Anhui, China. Email address: ynyang@ustc.edu.cn
} 
The run shock model has been widely applied to handle real-life problems. Usually, the system under a run shock model breaks down if there is a sequence of shocks above a predetermined threshold. However, in some practical problems, a single threshold is not enough to distinguish the severity of damage caused by the shocks. Goonewardene et al. [6] studied an applied problem in the healthcare management area, where prostate-specific antigen levels in the treatment of patients was divided into three levels with two thresholds. The choice of therapy was determined by the type of situation. This example reveals the significance of using more than one threshold to arrive at a precise classification of the shock magnitude. With this area as our motivation, we develop a generalized run shock model with two thresholds and study the reliability of systems under the proposed model.

If the number of consecutive shocks above a given threshold is considered as a random variable, then its movement can be treated as a discrete-time Markov process. According to the definition of the run shock model, this Markov chain will have finite transient states and one absorbing state. Similarly, in the generalized run shock model, consider a binary variable which counts the number of consecutive shocks above two thresholds. The state of this Markov chain will change when a shock occurs, and the system breaks down whenever one of the two variables exceeds its upper limit, regardless of the value of the other one. Thus, the movement of this binary variable also follows a discrete-time Markov process, which has finite transient states and several absorbing states. A feasible way to simplify the model is to merge all the absorbing states into a one-dimensional state. A phase-type distribution represents the time until absorption in a finite-state Markov chain with several transient states and one absorbing state. As an immediate consequence, the total number of shocks until system failure will follow a discrete phase-type distribution. We refer the reader to Eryilmaz [2], [5] for further examples where a phase-type distribution is applied to a discrete-time risk model.

Except for the number of shocks until failure, the lifetime of system is also determined by the inter-arrival time between shocks, in other words, the residual time in each state of the Markov process. Usually, they are assumed to follow a normal distribution, exponential distribution, or other typical distribution. However, the remarkable properties of these precise assumptions can lead to poor applicability. As an example, the memoryless property of an exponential distribution means that the system behaves as if it had just started up at any time point and its mean residual lifetime is not affected by how long the system has been in the working state, which is sometimes unreasonable [13].

The time until absorption in a continuous-time Markov chain follows a continuous phase-type distribution. It is an extension of an exponential distribution, using matrix form parameters as a substitute for numerical parameters, so it keeps the convenience of an exponential distribution in computation. Besides, any nonnegative distribution can be approximated arbitrarily closely by a phase-type distribution [12]. To sum up, a phase-type distribution has strong versatility to satisfy the requirements of modelling general phenomena. Eryilmaz [3], [4] introduced in detail the properties of a continuous phase-type distribution and its application. Following his approach, we assume that the inter-arrival time between shocks follows a continuous phase-type distribution. Configuration of the distribution parameters will be discussed later.

The paper is organized as follows. Section 2 involves the statistical preliminaries and description of our problem. A detailed analysis of the reliability properties of the system, including the total number of shocks until failure, mean time to failure, and mean residual lifetime will be conducted in Section 3. Considering that the occurrence frequency of shocks may increase along with the deterioration of the system state, we propose an extension to our model, so that different distributions can be used for modelling the inter-arrival time between 
shocks under different system states. We present the extended model in Section 4, together with a numerical example for illustrative purpose. In Section 5 we conclude the paper and discuss potential future research topics.

\section{Description of the system}

Assume that a system is subject to random shocks over time. Define two critical levels $c_{1}<c_{2}$. For given positive integers $k_{1}$ and $k_{2}$, the system is assumed to fail if at least $k_{1}$ consecutive shocks with magnitude above $c_{1}$ or $k_{2}$ consecutive shocks with magnitude above $c_{2}$ occur. It is obvious that $k_{1}>k_{2}$. Let $X_{i}$ denote the magnitude of the $i$ th shock, and $Y_{i}$ denote the inter-arrival time between the $(i-1)$ th and $i$ th shock. Assume that $X_{i}$ are independent and identically distributed (i.i.d.), and fall into the three intervals divided by the two thresholds with probabilities $p_{1}=\mathbb{P}\left(X_{i} \leq c_{1}\right), p_{2}=\mathbb{P}\left(c_{1}<X_{i} \leq c_{2}\right)$, and $p_{3}=\mathbb{P}\left(X_{i}>c_{2}\right)$.

As we have mentioned before, the system can be regarded as a discrete-time Markov chain with finite transient states and one absorption state. That is, the state of the system after a shock is determined by the state of the system before this shock, and is independent of previous shocks. Let $(s, t)$ represent one of the transient states when there are $s$ consecutive shocks with magnitude above $c_{1}$ and $t$ consecutive shocks with magnitude above $c_{2}$. Again, we always have $s \geq t$. We now provide an example for illustration.

Example 1. Consider the case when $k_{1}=2$ and $k_{2}=1$. The state space consists of five states: $\{(0,0),(1,0),(2,0),(1,1),(2,1)\}$, denoted by state $\{1,2,3,4,5\}$. This is an absorbing Markov chain with two transient and three absorbing states. Its transition probability matrix is

$$
\boldsymbol{P}=\left[\begin{array}{ccccc}
p_{1} & p_{2} & 0 & p_{3} & 0 \\
p_{1} & 0 & p_{2} & 0 & p_{3} \\
0 & 0 & 1 & 0 & 0 \\
0 & 0 & 0 & 1 & 0 \\
0 & 0 & 0 & 0 & 1
\end{array}\right]
$$

If we regard the set of state $\{3,4,5\}$ as a one-dimensional absorption state of state $\{1\}$ and state $\{2\}$, then the new transition probability matrix can be represented as

$$
\boldsymbol{P}^{\prime}=\left[\begin{array}{ccc}
p_{1} & p_{2} & p_{3} \\
p_{1} & 0 & p_{2}+p_{3} \\
0 & 0 & 1
\end{array}\right]
$$

The distribution of a discrete random variable $N$ is said to be phase-type if it is the distribution of the time until absorption in an discrete-time absorbing Markov chain. Consider a discretetime Markov chain with $t$ transient states and one absorbing state. It has a transition probability matrix of dimension $t+1$ and of the form

$$
\boldsymbol{P}=\left[\begin{array}{cc}
\boldsymbol{A} & \boldsymbol{A}^{0} \\
\mathbf{0} & \mathbf{1}
\end{array}\right],
$$

where $\boldsymbol{A}^{0}=(\boldsymbol{I}-\boldsymbol{A}) \boldsymbol{e}^{\top}$ with $\boldsymbol{e}$ a row vector with all elements equal to 1 and $\boldsymbol{I}$ the identity matrix. The initial probability vector is denoted by $\left(\boldsymbol{\alpha}, \alpha_{t+1}\right)$. Then the pair $(\boldsymbol{\alpha}, \boldsymbol{A})$ is called a representation for the derivative phase-type distribution and the time until absorption in this Markov chain follows $\mathrm{PH}_{\mathrm{d}}(\boldsymbol{\alpha}, \boldsymbol{A})$. 
In the example, the number of shocks until system failure follows a discrete phase-type distribution with representation

$$
\left((1,0),\left[\begin{array}{cc}
p_{1} & p_{2} \\
p_{1} & 0
\end{array}\right]\right)
$$

and probability mass function

$$
f(x)=\boldsymbol{\alpha} \boldsymbol{A}^{x-1} \boldsymbol{A}^{0}=\left[\begin{array}{ll}
1 & 0
\end{array}\right]\left[\begin{array}{cc}
p_{1} & p_{2} \\
p_{1} & 0
\end{array}\right]^{x-1}\left[\begin{array}{c}
p_{3} \\
p_{2}+p_{3}
\end{array}\right]
$$

To summarize, the total number of shocks until system failure in such a generalized run shock model will always follow a discrete phase-type distribution. If the inter-arrival time between shocks $Y_{i}$ is also assumed to follow a continuous phase-type distribution, then the lifetime of the system can be represented by the summation of several phase-type random variables, which is also phase-type according to its closure property under addition [9]. A representation for the summation of the $Y_{i}$ is possible if they are i.i.d. However, in the current article, we consider the approach of adapting a discrete-time Markov chain into a continuous chain to model a continuous variable, so that the system lifetime can be obtained directly, instead of computing the summation of several variables. This constitutes a significant difference between the methodology in this article and previous works.

\section{Analytical properties of the system}

In this section we study the total number of shocks until system failure, and then explain the major reliability characteristics and their computation methods.

\subsection{Total number of shocks until system failure}

Following the approach in Section 2, let a binary random variable $(s, t)$ represent the status when there are $s$ consecutive shocks with magnitude above $c_{1}$ and $t$ consecutive shocks with magnitude above $c_{2}$. Since the state of $(s, t)$ after a shock is determined by its state before the shock, and is independent of previous shocks, then the movement of $(s, t)$ is a Markov chain. It is not difficult to find that the value of $s$ and $t$ will either increase by one unit, or reset to zero. Besides, whenever the value of $t$ increases, the value of $s$ will increase simultaneously. To sum up, it will make the transition into state $(0,0)$ with probability $p_{1}$, into state $(s+1, t)$ with probability $p_{2}$, and into state $(s+1, t+1)$ with probability $p_{3}$. Furthermore, whenever the value of $s$ reaches $k_{1}$ or $t$ reaches $k_{2}$, it turns into an absorbing state, and will no longer make the transition to other states. In Figure 1 we present a representation of the transition rule of this chain.

We can conclude from Figure 1 that the Markov chain has $n=\frac{1}{2} k_{2}\left(2 k_{1}-k_{2}+1\right)$ transient states and $k_{1}+1$ absorption states. For the convenience of notation, the absorbing states are treated as an indivisible whole, while positive integers are allocated to the transient states in ascending order and by row. For example, state $(0,0)$ is denoted by state $\{1\}$, state $(1,0)$ is denoted by state $\{2\}$, and so on. Finally, the state $\left(k_{1}-1, k_{2}-1\right)$ is denoted by state $\{n\}$. Then the 
transition probability matrix between these transient states can be formulated as

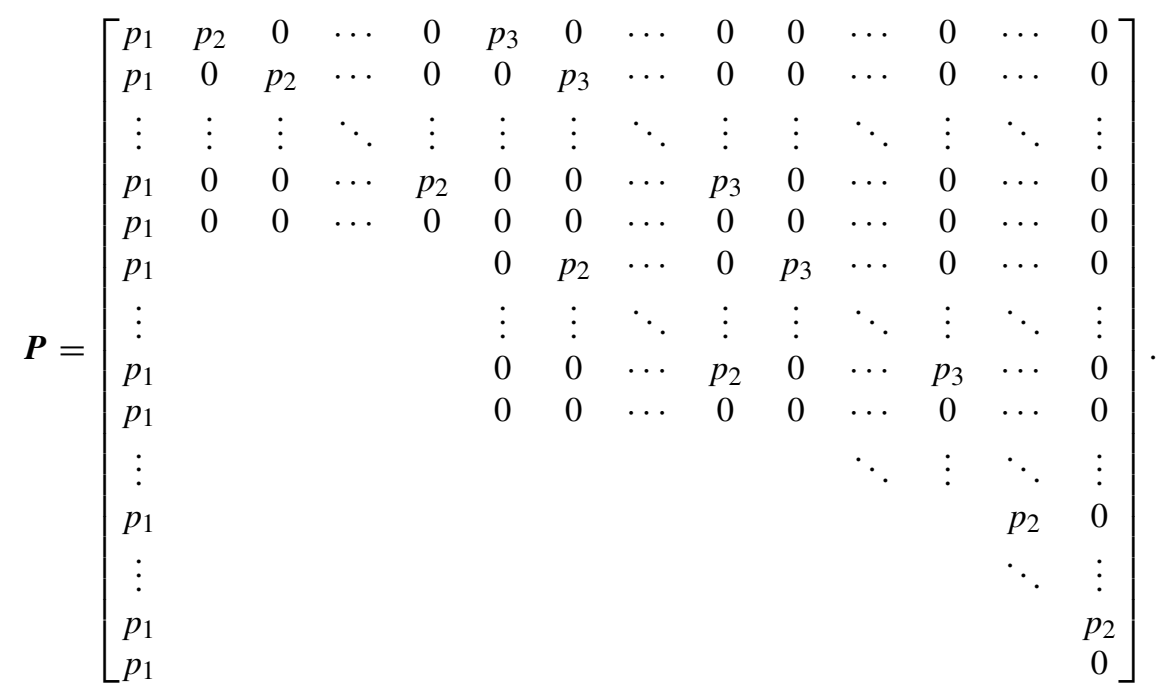

If we define three matrix types each with typical form

$$
\begin{gathered}
\boldsymbol{E}_{i}=\left[\begin{array}{cccc}
p_{1} & 0 & \cdots & 0 \\
p_{1} & 0 & \cdots & 0 \\
\vdots & \vdots & \ddots & \vdots \\
p_{1} & 0 & \cdots & 0
\end{array}\right]_{i \times k_{1}}, \quad \boldsymbol{S}_{i}=\left[\begin{array}{cccc}
0 & p_{2} & \cdots & 0 \\
0 & \vdots & \ddots & 0 \\
\vdots & 0 & \cdots & p_{2} \\
0 & 0 & \cdots & 0
\end{array}\right]_{i \times i} \\
\boldsymbol{T}_{i}=\left[\begin{array}{ccc}
p_{3} & \cdots & 0 \\
\vdots & \ddots & 0 \\
0 & \cdots & p_{3} \\
0 & \cdots & 0
\end{array}\right]_{i \times i-1},
\end{gathered}
$$

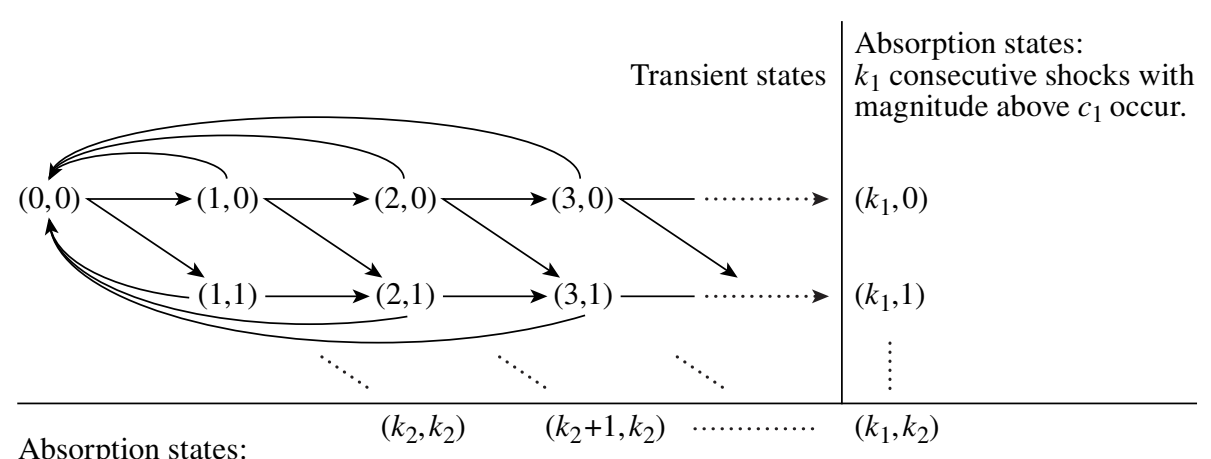

Absorption states:

$k_{2}$ consecutive shocks with magnitude above $c_{2}$ occur.

FIGURE 1: Transition rule. 
then the transition probability matrix $\boldsymbol{P}$ can be blocked into

$$
\left[\begin{array}{ccc}
\boldsymbol{P}_{1,1} & \cdots & \boldsymbol{P}_{1, k_{2}} \\
\vdots & \ddots & \vdots \\
\boldsymbol{P}_{k_{2}, 1} & \cdots & \boldsymbol{P}_{k_{2}, k_{2}}
\end{array}\right],
$$

where $\boldsymbol{P}_{i, i+1}=\boldsymbol{T}_{k_{1}-i+1}$ for any $i=1, \ldots, k_{2}-1, \boldsymbol{P}_{j, 1}=\boldsymbol{E}_{k_{1}-j+1}, \boldsymbol{P}_{j, j}=\boldsymbol{S}_{k_{1}-i+1}$ for any $j=2, \ldots, k_{2}$, and $\boldsymbol{P}_{i, j}=\mathbf{0}$ otherwise. In particular, for the first block $\boldsymbol{P}_{1,1}=\boldsymbol{E}_{k_{1}}+\boldsymbol{S}_{k_{1}}$. To summarize, $\boldsymbol{P}$ can be represented as

$$
\boldsymbol{P}=\left[\begin{array}{ccccc}
\boldsymbol{E}_{k_{1}}+\boldsymbol{S}_{k_{1}} & \boldsymbol{T}_{k_{1}} & & & \\
\boldsymbol{E}_{k_{1}-1} & \boldsymbol{S}_{k_{1}-1} & \boldsymbol{T}_{k_{1}-1} & & \\
\boldsymbol{E}_{k_{1}-2} & & \boldsymbol{S}_{k_{1}-2} & \ddots & \\
\vdots & & & \ddots & \boldsymbol{T}_{k_{1}-k_{2}+2} \\
\boldsymbol{E}_{k_{1}-k_{2}+1} & & & & \boldsymbol{S}_{k_{1}-k_{2}+1}
\end{array}\right] .
$$

According to the definition, the total number of shocks until system failure is the time until absorption in the above discrete-time Markov chain, which follows a discrete phase-type distribution $\mathrm{PH}_{\mathrm{d}}(\boldsymbol{p}, \boldsymbol{P})$, where $\boldsymbol{p}$ is the initial vector of the Markov chain and $\boldsymbol{P}$ is the transition probability matrix. When the system is unrepairable, it will always start working at the perfect working state. Thus, the initial vector is $\boldsymbol{p}=(1,0, \ldots, 0)$.

\subsection{Reliability characteristics of the system}

The lifetime of the system is determined jointly by the total number of shocks before system failure and the inter-arrival time between shocks, while the latter one should be a continuous random variable. Considering the versatility, we assume that the inter-arrival time between shocks follows a continuous phase-type distribution.

The distribution of a continuous random variable $X$ is said to be phase-type if it is the distribution of the time until absorption in a finite-state continuous-time Markov chain with $m$ transient states and one absorbing state. Assume the initial probability vector is $\left(\boldsymbol{\alpha}, \alpha_{t+1}\right)$, where $\alpha_{t+1}=1-\boldsymbol{\alpha} \boldsymbol{e}^{\top}$, and the transition rate matrix is

$$
\left[\begin{array}{cc}
\boldsymbol{A} & -\boldsymbol{A} \boldsymbol{e}^{\top} \\
\mathbf{0} & 0
\end{array}\right]
$$

then the random variable $X$ will have representation $\mathrm{PH}_{\mathrm{c}}(\boldsymbol{\alpha}, \boldsymbol{A})$. Here, $\boldsymbol{e}$ is a row vector with all elements equal to 1 , and its dimension depends on the left-multiplication matrix.

Assume that the inter-arrival time between shocks $Y_{i}$ are i.i.d., following a continuous phasetype distribution $\mathrm{PH}_{\mathrm{c}}(\boldsymbol{\alpha}, \boldsymbol{A})$, and there are $N$ shocks before system failure. Then the system lifetime can be calculated as $T=\sum_{i=1}^{N} Y_{i}$. He [9] proved a computational proposition for this type of summation.

Proposition 1. Assume that $Y_{1}, Y_{2}, \ldots$ are independent with $Y_{i} \sim \mathrm{PH}_{\mathrm{c}}(\boldsymbol{\alpha}, \boldsymbol{A})$, and, independently, $N \sim \mathrm{PH}_{\mathrm{d}}(\boldsymbol{p}, \boldsymbol{P})$. If $\boldsymbol{\alpha}$ and $\boldsymbol{p}$ are stochastic vectors, that is, $\boldsymbol{\alpha} \boldsymbol{e}^{\top}=1, \boldsymbol{p} \boldsymbol{e}^{\top}=1$, then

$$
\sum_{i=1}^{N} Y_{i} \sim \mathrm{PH}_{\mathrm{c}}\left(\boldsymbol{\alpha} \otimes \boldsymbol{p}, \boldsymbol{A} \otimes \boldsymbol{I}+\left(\boldsymbol{A}^{0} \boldsymbol{\alpha}\right) \otimes \boldsymbol{P}\right),
$$

where $\boldsymbol{A}^{0}=-\boldsymbol{A} \boldsymbol{e}^{\top}$ and $\otimes$ is the Kronecker product. 
It is obvious that if we denote $\boldsymbol{\beta}=\boldsymbol{\alpha} \otimes \boldsymbol{p}$ and $\boldsymbol{Q}=\boldsymbol{A} \otimes \boldsymbol{I}+\left(\boldsymbol{A}^{0} \boldsymbol{\alpha}\right) \otimes \boldsymbol{P}$, then the system lifetime will follow the $\mathrm{PH}$ distribution $\mathrm{PH}_{\mathrm{c}}(\boldsymbol{\beta}, \boldsymbol{Q})$. According to the well-known properties of the phase-type distribution, the distribution function of the system lifetime $T$ can be represented by

$$
F(t)=1-\boldsymbol{\beta} \exp \{\boldsymbol{Q} t\} \boldsymbol{e}^{\top}=1-\boldsymbol{\beta}\left(\sum_{n=0}^{\infty} \frac{x^{n}}{n !} \boldsymbol{Q}^{n}\right) \boldsymbol{e}^{\top}
$$

The system mean time to failure (applied to an unrepairable system) or mean time between failures (for a repairable system) can be computed from

$$
\mathbb{E}[T]=-\boldsymbol{\beta} \boldsymbol{Q}^{-1} \boldsymbol{e}^{\top}
$$

Besides, another important reliability characteristic is the mean residual lifetime function at a given time [3]. The reliability significance of this function is the average residual time until failure when the system is still working at time $t$. When compared with the average lifetime, this statistic is more flexible since it can be monitored throughout the working state. As He [9] pointed out, for any continuous $\mathrm{PH}$ random variable $X \sim \mathrm{PH}(\boldsymbol{\alpha}, \boldsymbol{A})$,

$$
\{X-t \mid X>t\} \sim \operatorname{PH}\left(\frac{\boldsymbol{\alpha} \exp \{\boldsymbol{A} t\}}{\boldsymbol{\alpha} \exp \{\boldsymbol{A} t\} \boldsymbol{e}^{\top}}, \boldsymbol{A}\right) .
$$

Thus, given that the system is still performing at any time point $t$, the mean residual lifetime of the system is

$$
\mathbb{E}[T-t \mid T>t]=-\frac{\boldsymbol{\beta} \exp \{\boldsymbol{Q} t\}}{\boldsymbol{\beta} \exp \{\boldsymbol{Q} t\} \boldsymbol{e}^{\top}} \boldsymbol{Q}^{-1} \boldsymbol{e}^{\top}
$$

\section{A Markovian approach for reliability assessment}

In Section 3 we proposed a reliability model for multi-state systems. However, the model is restricted to the assumption that the inter-arrival times between shocks are i.i.d. When dealing with real-world problems, many researchers have studied a type of system whose properties change along with system degradation; see [13], [15]-[17]. Inspired by their work, the underlying model can be adapted to the situation that the inter-arrival time between shocks follows a different distribution under a different system state. Since the system state makes a transition whenever a shock occurs, the inter-arrival time between shocks can also be interpreted as the residence time in the corresponding state.

Usually, a system is more likely to be affected by external factors when it is partially broken. In other words, the parameters should be configured to make the expected residence time in the partially working states be shorter than the perfect working state. In this section we provide a general method of constructing a continuous Markov chain to model the system lifetime.

\subsection{Phase-type representation of the system lifetime}

As mentioned before, the inter-arrival time of shocks can be regarded as the residence time. We follow the configuration in Section 3 that the system is modelled by a discrete Markov chain with $n$ different working states, and assume that the residence time in state $\{i\}$ follows the distribution $\operatorname{PH}\left(\boldsymbol{\alpha}_{i}, \boldsymbol{A}_{i}\right)$, where the dimension of matrix $\boldsymbol{A}_{i}$ is $d_{i}$. In other words, when the system remains in state $\{i\}$, the inter-arrival time between shocks will follow $\operatorname{PH}\left(\boldsymbol{\alpha}_{i}, \boldsymbol{A}_{i}\right)$.

From the definition, $\mathrm{PH}\left(\boldsymbol{\alpha}_{i}, \boldsymbol{A}_{i}\right)$ is the distribution of absorption time in a continuous Markov chain with $d_{i}$ transition states. Thus, the system state $\{i\}$ can be further divided into $d_{i}$ substates. 
As a result, the system lifetime can be modelled by a continuous Markov chain with $\sum_{i=1}^{n} d_{i}$ transient states in total, each of these states is a substate of one of the system states. The transition rule in this chain can be summarized as follows.

Rule 1 . When the system stays in state $\{i\}$, the system must stay in one of its $d_{i}$ substates.

Rule 2. During the residence in state $\{i\}$, transition among its $d_{i}$ substates may happen according to the transition rate matrix $\boldsymbol{A}_{i}$.

Rule 3. When the system leaves state $\{i\}$ and transfers into state $\{j\}$, the current substate will transfer into one of the $d_{j}$ substates. The rate of leaving the current substate is equal to the corresponding element in $\boldsymbol{A}_{i}^{0}=-\boldsymbol{A}_{i} \boldsymbol{e}^{\top}$, and the probability of transiting into each of the $d_{j}$ substates is equal to the corresponding element in the initial probability vector $\boldsymbol{\alpha}_{j}$.

In order to obtain the phase-type representation of the system lifetime, we have to compute the transition rate matrix between the substates. Whenever a substate $a$ transfers to substate $b$, the transition will be classified as one of three situations, according to the adscription of both substates.

Situation 1. When substate $a$ belongs to state $\{1\}$, and substate $b$ also belongs to state $\{1\}$, there exist two possibilities:

- the transition occurs among the $d_{1}$ substates according to rule 2;

- the system leaves state $\{1\}$ but transfers into state $\{1\}$ again according to rule 3 . The transition probability from state $\{1\}$ to state $\{1\}$ is $p_{1}$. In Figure 2 we present an illustration of the transition process.

From the figure, the transition rate under the first case is $\boldsymbol{A}_{1}$, while the transition rate under the second case is $p_{1} \boldsymbol{A}_{1}^{0} \boldsymbol{\alpha}_{1}$. As a result, the complete transition rate matrix is $\boldsymbol{A}_{1}+p_{1} \boldsymbol{A}_{1}^{0} \boldsymbol{\alpha}_{1}$.

Situation 2. When substate $a$ belongs to state $\{i\}$ (with $i \neq 1$ ), and substate $b$ also belongs to state $\{i\}$, there exists only one possibility, because when $i \neq 1$, the system state will never remain in place after a shock occurs. Thus, the transition occurs among the $d_{i}$ substates according to rule 2. In Figure 3 we present an illustration of the transition process. From the figure, the transition rate is $\boldsymbol{A}_{i}$.

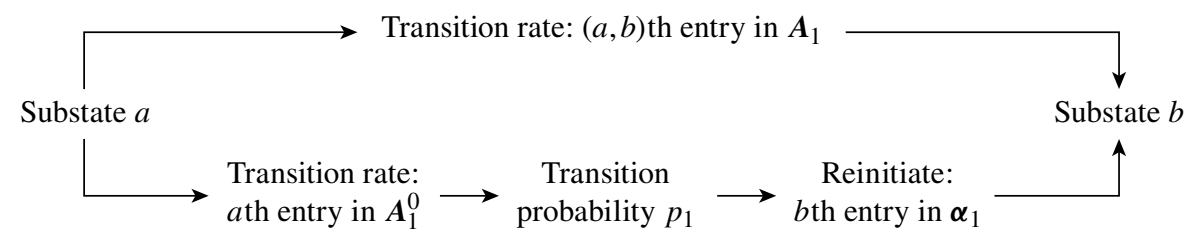

FIGURE 2: Transition from state $\{1\}$ to state $\{1\}$.

Substate $a \longrightarrow$ Transition rate: $(a, b)$ th entry in $\boldsymbol{A}_{i}$ Substate $b$

FIGURE 3: Transition from state $\{i\}$ to state $\{i\}$ (with $i \neq 1)$. 
Substate $a \longrightarrow \begin{aligned} & \text { Transition rate: } \\ & \text { th entry in } \boldsymbol{A}_{i}^{0}\end{aligned} \longrightarrow \begin{gathered}\text { Transition } \\ \text { probability } p_{i j}\end{gathered} \longrightarrow \begin{gathered}\text { Reinitiate: } \\ b \text { th entry in } \boldsymbol{\alpha}_{j}\end{gathered} \longrightarrow$ Substate $b$

FIGURE 4: Transition from state $\{i\}$ to $\operatorname{state}\{j\}$ (with $i \neq j$ ).

Situation 3. When substate $a$ belongs to state $\{i\}$, and substate $b$ belongs to state $\{j\}$ (with $i \neq j$ ), the system leaves system state $\{i\}$, and transfers into system state $\{j\}$ according to rule 3 . The transition probability from state $\{i\}$ to $\operatorname{state}\{j\}$ is $p_{i j}$. In Figure 4 we present an illustration of the transition process. From the figure, the transition rate has the form $p_{i j} \boldsymbol{A}_{i}^{0} \boldsymbol{\alpha}_{j}$.

To summarize, the system's transition rate matrix between all the transient states is

$$
\boldsymbol{Q}=\left[\begin{array}{cccc}
\boldsymbol{A}_{1}+p_{1} \boldsymbol{A}_{1}^{0} \boldsymbol{\alpha}_{1} & p_{12} \boldsymbol{A}_{1}^{0} \boldsymbol{\alpha}_{2} & \cdots & p_{1 n} \boldsymbol{A}_{1}^{0} \boldsymbol{\alpha}_{n} \\
p_{21} \boldsymbol{A}_{2}^{0} \boldsymbol{\alpha}_{1} & \boldsymbol{A}_{2} & \cdots & p_{2 n} \boldsymbol{A}_{2}^{0} \boldsymbol{\alpha}_{n} \\
\vdots & \vdots & \ddots & \vdots \\
p_{n 1} \boldsymbol{A}_{n}^{0} \boldsymbol{\alpha}_{1} & p_{n 2} \boldsymbol{A}_{n}^{0} \boldsymbol{\alpha}_{2} & \cdots & \boldsymbol{A}_{n}
\end{array}\right]
$$

where $p_{i j}$ is the element on the $i$ th row and $j$ th column of the transition probability matrix $\boldsymbol{P}$, which can be computed from (1).

To sum up, the lifetime of the system follows a continuous phase-type distribution with PH-generator $\boldsymbol{Q}$, while the substochastic vector can be generated from the initial probability vector. When the system is unrepairable, the chain will always start at the perfect working state, so its initial vector is $\boldsymbol{\beta}=\left(\boldsymbol{\alpha}_{1} / \boldsymbol{\alpha}_{1} \boldsymbol{e}^{\top}, \mathbf{0}\right)$. Once $\boldsymbol{\beta}$ and $\boldsymbol{Q}$ are determined, the reliability characteristics of the system can be computed from (3)-(5).

\subsection{Numerical example}

In this subsection we conduct a real-data analysis in order to illustrate our results. We continue with the example in Section 2, and assume that the two thresholds are determined so that the occurrence probability of a shock with magnitude greater than $c_{1}$ is 0.5 , while the probability of a shock greater than $c_{2}$ is 0.2 . In other words, we have $p_{1}=0.5$ and $p_{2}=0.3$ in the example. We can easily compute that the number of shocks until system failure follows a discrete phase-type distribution

$$
\mathrm{PH}_{\mathrm{d}}\left((1,0),\left[\begin{array}{cc}
0.5 & 0.3 \\
0.5 & 0
\end{array}\right]\right) .
$$

Besides, we consider the case that the inter-arrival time between shocks under a perfect working state follows a continuous phase-type distribution

$$
\mathrm{PH}_{\mathrm{c}}\left((0.5,0.5),\left[\begin{array}{cc}
-2 & 2 \\
0 & -2
\end{array}\right]\right),
$$

and its expectation is 0.75 units of time. We check the performance of our model by comparing three types of system.

Type 1 system. The system properties are never affected by external shocks. In other words, the distribution of inter-arrival times between shocks is the same after the system degrades.

Type 2 system. The system properties are affected after degradation caused by external shocks. The distribution of inter-arrival times between shocks under state $\{2\}$ (state $(1,0)$ ) changes to another continuous $\mathrm{PH}$ distribution

$$
\mathrm{PH}_{\mathrm{c}}\left((0.7,0.3),\left[\begin{array}{cc}
-1.667 & 0 \\
0.625 & -1.25
\end{array}\right]\right),
$$

but this distribution has the same expectation as the previous one. 
Type 3 system. The system properties are affected after degradation caused by external shocks. The distribution of inter-arrival times between shocks under state $\{2\}(\operatorname{state}(1,0))$ is changed to another continuous $\mathrm{PH}$ distribution

$$
\mathrm{PH}_{\mathrm{c}}\left((0.8,0.2),\left[\begin{array}{cc}
-8 & 4 \\
12 & -16
\end{array}\right]\right),
$$

and the expectation of this distribution is decreased to 0.25 units of time. In other words, the system becomes more vulnerable after a degradation, with the occurrence of a new shock tending to arrive earlier than before.

It is easy to see that the lifetime of a type 1 system is a summation of i.i.d. continuous phasetype variables, which can be computed from (2), while the lifetimes of type 2 and type 3 systems can be calculated from (6). However, the computation in (2) is complicated as it includes a high-dimensional matrix product, and the assumption that all the variables in the summation must be i.i.d. will restrict its application. The type 2 and type 3 systems are more practical.

The analytical solutions can be expressed as follows:

a type 1 system lifetime follows

$$
\mathrm{PH}_{\mathrm{c}}\left((0.5,0,0.5,0),\left[\begin{array}{cccc}
-2 & 0 & 2 & 0 \\
0 & -2 & 0 & 2 \\
0.5 & 0.3 & -1.5 & 0.3 \\
0.5 & 0 & 0.5 & -2
\end{array}\right]\right)
$$

a type 2 system lifetime follows

$$
\mathrm{PH}_{\mathrm{c}}\left((0.5,0.5,0,0),\left[\begin{array}{cccc}
-2 & 2 & 0 & 0 \\
0.5 & -1.5 & 0.42 & 0.18 \\
0.42 & 0.42 & -1.67 & 0 \\
0.16 & 0.16 & 0.63 & -1.25
\end{array}\right]\right)
$$

a type 3 system lifetime follows

$$
\mathrm{PH}_{\mathrm{c}}\left((0.5,0.5,0,0),\left[\begin{array}{cccc}
-2 & 2 & 0 & 0 \\
0.5 & -1.5 & 0.48 & 0.12 \\
1 & 1 & -8 & 4 \\
1 & 1 & 12 & -16
\end{array}\right]\right) .
$$

In Figure 5 we present the reliability functions of the three types of system.

We can directly conclude from the graph that although the phase-type representation of type 1 and type 2 system lifetimes is different, there is no difference between them in nature. It reveals the feasibility of calculating the system lifetime through the properties of a continuous Markov chain, which is equivalent to the summation of several random variables, but much easier in computational terms. Besides, the reliability of a vulnerable system is lower than a stable one, which also coincides with what we expected.

\section{Conclusions and discussion}

In this paper we introduced a generalized run shock model. The main motivation of this work is to broaden the application field of shock models in real-world problems. A twothreshold criteria for shock magnitude was considered. The properties of Markov chains 


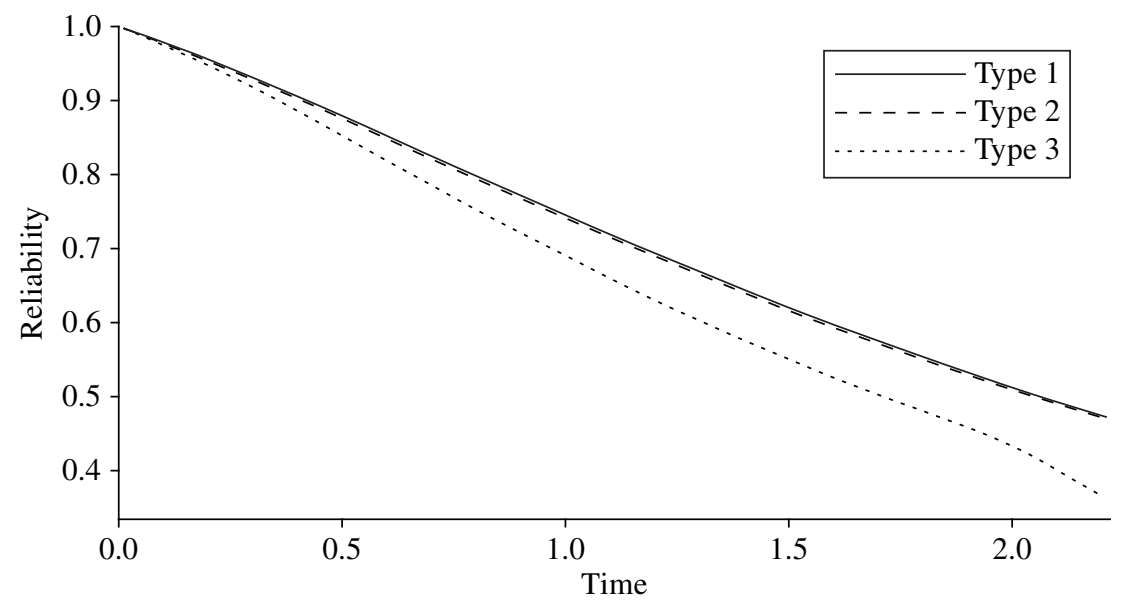

FIGURE 5: Reliability functions for the three types of system.

and their derivative probability distributions allowed a highly versatile model construction. Computation formulas for several important system reliability characteristics were presented. Our numerical example illustrated the research value of our work by revealing favourably computational convenience.

When applying the model to repairable systems, we choose the initial probability vector according to the maintenance policy. For example, assume that the system is restored to working state $i$ with probability $\pi_{i}(i=1, \ldots, n)$ after repairing, then the initial vector is

$$
\boldsymbol{\beta}=\left(\pi_{1} \frac{\boldsymbol{\alpha}_{1}}{\boldsymbol{\alpha}_{1} \boldsymbol{e}^{\top}}, \ldots, \pi_{n} \frac{\boldsymbol{\alpha}_{n}}{\boldsymbol{\alpha}_{n} \boldsymbol{e}^{\top}}\right) .
$$

The maintenance policy is beyond the scope of our research, so we are concerned only with unrepairable systems in this paper, as is common practice.

For a potential extension of this work, we can consider a further generalized run shock model with more than one failure state, as modern systems with several failure states can start recovery before a complete failure. Besides, there is a pressing need for a clear configuration method for the phase-type parameters for the residence time distribution. Furthermore, different types of maintenance policy, such as replacement, minimal repair, or imperfect maintenance may warrant investigation. Finally, other types of shock model should be considered, so that special mixed models can be designed for typical problems. We leave these as future research topics.

\section{References}

[1] Cha, J. H. and Finkelstein, M. (2011). On new classes of extreme shock models and some generalizations. J. Appl. Prob. 48, 258-270.

[2] Eryilmaz, S. (2013). On the lifetime behavior of a discrete time shock model. J. Comput. Appl. Math. 237, 384-388.

[3] Eryilmaz, S. (2015). Assessment of a multi-state system under a shock model. Appl. Math. Comput. 269, 1-8.

[4] Eryilmaz, S. (2015). Dynamic assessment of multi-state systems using phase-type modeling. Reliab. Eng. Syst. Safety 140, 71-77.

[5] Eryilmaz, S. (2016). Compound Markov negative binomial distribution. J. Comput. Appl. Math. 292, 1-6.

[6] Goonewardene, S. S., Phull, J. S. B. A. and Persad, R. A. (2014). Interpretation of PSA levels after radical therapy for prostate cancer. Tr. Urol. Mens Health 5, 30-34.

[7] Gut, A. (1990). Cumulative shock models. Adv. Appl. Prob. 22, 504-507. 
[8] Gut, A. (2001). Mixed shock models. Bernoulli 7, 541-555.

[9] HE, Q.-M. (2014). Fundamentals of Matrix-Analytic Methods. Springer, New York.

[10] LIN, Y. H., LI, Y. F. AND ZIO, E. (2016). Reliability assessment of systems subject to dependent degradation processes and random shocks. IIE Trans. 48, 1072-1085.

[11] Mallor, F., Omey, E. And Santos, J. (2006). Asymptotic results for a run and cumulative mixed shock model. J. Math. Sci. 138, 5410-5414.

[12] Montoro-Cazorla, D. And Pérez-Ocón, R. (2006). Replacement times and costs in a degrading system with several types of failure: the case of phase-type holding times. Europ. J. Operat. Res. 175, 1193-1209.

[13] Peng, D., Fang, L. And Tong, C. (2013). A multi-state reliability analysis of single-component repairable system based on phase-type distribution. In Proc. Int. Conf. Management Science and Engineering. IEEE, Piscataway, NJ, pp. 496-501.

[14] Peng, R., Zhai, Q. Q. And Levitin, G. (2016). Defending a single object against an attacker trying to detect a subset of false targets. Reliab. Eng. System Safety 149, 137-147.

[15] Segovia, M. C. and Labeau, P. E. (2013). Reliability of a multi-state system subject to shocks using phase-type distributions. Appl. Math. Model. 37, 4883-4904.

[16] Shrestha, A., XING, L. AND DAI, Y. (2011). Reliability analysis of multistate phased-mission systems with unordered and ordered states. IEEE Trans. Syst. Man. Cyb. A 41, 625-636.

[17] YINGKUI, G. AND Jing, L. (2012). Multi-state system reliability: a new and systematic review. Procedia Engineer. 29, 531-536.

[18] Zhou, X., Wu, C. Y, LI, Y. ANd XI, L. (2016). A preventive maintenance model for leased equipment subject to internal degradation and external shock damage. Reliab. Eng. Syst. Safety 154, 1-7. 